\title{
Wilhelm Wien's Photons Creating the BohEmian Pilot Wave for the Guiding of the Individual Huygens - de Broglie Particles on the Helical Path Governed by the Newton - Bohm Evolute (the Bohmian Pilot Wave) through the Young - Feynman Double - Slit Barrier. Wilhelm Wien's Photons in the Mach - Zehnder Interferometer (20.08.2019)
}

\author{
Jiř́ Stávek ${ }^{1}$ \\ ${ }^{1}$ Bazovského, Prague, Czech Republic \\ Correspondence: Jiř́i Stávek, Bazovského 1228, 16300 Prague, Czech Republic. E-mail: stavek.jiri@seznam.cz \\ Received: August 15, 2019 \\ Accepted: August 29, 2019 \\ Online Published: September 30, 2019 \\ doi:10.5539/apr.v1 $1 \mathrm{n} 5 \mathrm{p} 10$ \\ URL: http://dx.doi.org/10.5539/apr.v11n5p10
}

\begin{abstract}
In our approach we have combined knowledge of Old Masters (working in this field before the year 1905), New Masters (working in this field after the year 1905) and Dissidents under the guidance of Louis de Broglie and David Bohm. In our model the quantum particle is represented as the Huygens-de Broglie's particle on the helical path (full wave) guided by the Newton-Bohm entangled helical evolute (Bohmian Pilot Wave). These individual Huygens - de Broglie particles in the Young - Feynman double - slit experiment react with Wilhelm Wien's photons that are always present inside of the apparatus (Wien's displacement law). Wilhelm Wien's photons form collectively the Wien filter guiding the Huygens - de Broglie particles through the double - slit barrier towards a detector (BohEmian Pilot Wave). The interplay of those events creates the observed interference pattern. In the very well-known formula describing the intensity of double-slit diffraction patterns we have newly introduced the concept curvature $\kappa$ of the Huygens - de Broglie particle and thus giving a physical interpretation for the Newton - Bohm guiding wave (the Bohmian Pilot Wave): for photons $\kappa=\pi / \lambda$, for electrons $\kappa=2 \pi / \lambda$. Moreover, we have introduced into that formula the expression $\lambda_{\max }$ from the Wien's displacement law to describe geometry of the double - slit barrier. We propose to modify the value $\lambda_{\max }$ by the change of the system temperature. There is a second experimental possibility - we can insert into those slits filters to remove Wien's photons while the Huygens - de Broglie particles continue towards a detector - we should observe the particle behavior. The similar situation might occur in the Mach - Zehnder interferometer. In this case the individual Huygens - de Broglie particle reacts in the first beam splitter with the Wien photon: the Huygens - de Broglie particle goes through one path while the Wien photon goes through the second path. In the second beam splitter they interact again and create the interference pattern on one detector. We can experimentally modify the resulting interference pattern in the Mach - Zehnder interferometer - by the temperature change of the system or by inserting filters to remove Wien's photons from one or both paths. Can it be that Nature cleverly creates those interference patterns while the Bohmian pilot wave and the BohEmian pilot wave are hidden in plain sight? We want to pass this concept into the hands of Readers of this Journal better educated in the Mathematics and Physics.
\end{abstract}

Keywords: Huygens-de Broglie Particle on the Helical Path, Newton-Bohm Entangled Helical Evolute, Curvature of Helix, Bohmian Pilot Wave, Wien's Displacement Law, Wien's Photons, Wien Filter, BohEmian Pilot Wave, Filters to Remove Wien's photons

\section{Introduction}

The famous quote of Heraclitus "Nature loves to hide" was described in details by Pierre Hadot in 2008. Hadot in his valuable book gives us many examples how Nature protects Her Secrets. In several situations the enormous research of many generations is strongly needed before the right "recipe" unlocking the True reality can be found. Johann Wolfgang Goethe remarked to our research: "Nature does not suffer Her veil to be taken from Her, and what She does not choose to reveal to the spirit, thou wilt wrest from Her by levers and screws." 
In our model of the photon we have found that Nature could keep Her Secrets hidden in plain sight and thus can perfectly document Her Top Art of Hiding.

Cylindrical Helix represents one of the most fascinating curves in Nature and Science and is among the oldest curves. Cylindrical Helix belongs to the Treasure of Geometry. The Cylindrical Helix seem to have been discovered and thoroughly studied by Apollonius of Perga (The Great Geometer) and his scholars as e.g. Geminus of Rhodes. Cylindrical helix is composed from two motions - circular and translational. Heron discovered the cylindrical helix construction by triangle wrapping that gives to us a deeper view in the properties of the elastic helical WAVE. For the details of the Heron's construction see P. Mancosu and A. Arana (2010).

The model of the double helix for the description of the photon wave appeared many times in the so-called dissident literature (outside of the mainstream literature). There are known many proposals for this double helix composition. Louis de Broglie proposed at the 1927 Solvay Conference his model of the full wave and the pilot guiding wave but could not give a deeper physical interpretation of his concept. Later Louis de Broglie (1939) proposed two component model of the photon. (Many modern Dissidents continue to develop this double-helix model of the photon where both helical paths are occupied by particles). In 1952 David Bohm rediscovered this pilot wave model and developed it as the de Broglie - Bohm theory. Since that time the concept of empty guiding waves remains still open and has been waiting for the physical interpretation. See J.S. Bell in 1992, L. Hardy in 1992, P.J. Lewis in 2007, W. Seager in 2018, and many others.

Albert Einstein called these empty waves in the de Broglie-Bohm theory as the ghost waves ("Gespensterfelder", ghost fields) and the de Broglie - Bohmian concept as "too cheap". Are we able to find a physical meaning for those empty waves? In such case we might express here the old Bohemian proverb "Salt above Gold" as "Photons above Gold".

In order to achieve our target we have combined knowledge of Old Masters (working in this field before the year 1905), New Masters (working in this field after the year 1905), and Dissidents working on the double helix model of the photon for many years.

(We are aware of the famous quote of Richard Feynman: "We choose to examine a phenomenon (the double-slit experiment) which is impossible, absolutely impossible, to explain in any classical way, and which is in the heart of quantum mechanics.")

\section{Inspirations from Old Masters, New Masters, and Dissidents}

We were inspired by many Great Researchers working in this field for generations. Some of those Masters with their ideas are given in the Table 1. Based on their knowledge we can model the photon as a dyon travelling on the helical path.

Table 1. Inspirations from Great Masters to formulate a model for photon

FULL WAVE: Huygens - de Broglie particle on the helical path

EMPTY WAVE: Newton - Bohm entangled guiding helical evolute

H.A. Lorentz acting force of photons and reacting force of fermions

Super-elasticity of photon WAVES - Doppler's super-elasticity, Zwicky's super-elasticity

Dirac's quantum of magnetic flux where $\alpha$ is the helical angle

$\Theta_{D}=\frac{h}{e}=\frac{h}{e}(\cos \alpha)^{2}+\frac{h}{e}(\sin \alpha)^{2}$

Superconducting magnetic flux for $\alpha=45^{\circ}$

$\Theta_{0}=\frac{h}{2 e}$

Model of quarks by Gell- Mann and Zweig

Models of dyons by Schwinger

Model of dyons by Witten 


$$
\frac{e \theta}{2 \pi} \quad \text { for } \theta= \pm \pi \quad \pm \frac{e}{2}
$$

Schwinger - Zwanziger condition

$$
\begin{aligned}
& e_{1} g_{2}-e_{2} g_{1}=n h \\
& \frac{e}{2} \frac{h}{e}-\left(-\frac{e}{2}\right) \frac{h}{e}=h
\end{aligned}
$$

Polchinski: "The existence of monopoles is one of the safest bets that one can make about physics not yet seen".

This model of the photon particle as a dyon with the double helix structure: Huygens - de Broglie particle on the helical path guiding by the Newton - Bohm helical evolute can start a new view on some effects. With this new model of photon (dyon) we can newly describe some phenomena: e.g., the quantum of magnetic flux in superconductors, the events in the Young - Feynman experiment, the events in the Mach - Zehnder interferometer, etc.

\section{Wien's Displacement Law}

Wilhelm Wien derived in 1893 his famous Wien's displacement law: the black body radiation curve for different temperatures will peak at different wavelengths that are inversely proportional to the temperature:

Table 2. Wien's displacement law

$$
\begin{aligned}
& \text { Wien's Displacement Law } \\
& \lambda_{\max }=\frac{h c}{x} \frac{1}{k T} \approx \frac{2.898^{*} 10^{6} \mathrm{nmK}}{T} \\
& \lambda_{\max } \text { for } 300 K \approx 10 \mu \mathrm{m}
\end{aligned}
$$

Wien's photons are always present in our apparatuses - can they participate in the development of interference structures in the Young - Feynman double - slit experiment and in the Mach - Zehnder interferometer?

\section{Wien Filter in the Young - Feynman Double - Slit Experiment (BohEmian Pilot Wave)}

Wilhelm Wien in 1898 invented a device consisting of perpendicular electric and magnetic fields that can be used as a velocity filter for charged particles. See, e.g. Franz Hasselbach in 1992, Karl Wien in 1999, E. Plies et al. in 2011.

In our model we assume that Wien's photons (dyons) (Wien replacement law) are always present inside the double-slit apparatus and interact with individual Huygens - de Broglie particles as the Wien Filter. This interaction between Wien's photons and Huygens - de Broglie particles leads to the observed interference patterns. The collective of self-organized Wien's photons guiding the Huygens - de Broglie particle will be termed as the BohEmian Pilot Wave.

We can experimentally observe the influence of temperature $\left(\lambda_{\max }\right)$ on the resulting interference patterns. There is another experimental possibility to insert filters into the slits to selectively remove Wien's photons guiding those Huygens - de Broglie particles. 


\section{Young - Feynman Double - Slit Experiment with Individual Photons}

There were done numerous experiments to observe the pattern formation with individual photons below the double - slit barrier. See the References.

In the Table 2 is the traditional formula for the calculation of the intensity of double slit patterns for photons: $I$ is the intensity at the angle $\theta, I_{0}$ is the maximum intensity, $d$ is the separation between slits, $a$ is the slit width, $\lambda$ is the pitch of the photon double helix creating the interference structure.

We have modified this Formula in two steps:

1) We have "discovered" the hidden expression for the photon helical curvature $\kappa=\pi / \lambda$ - this is the hidden Bohmian pilot wave for photons.

2) We have described the separation between slits $d$ as $d=D \lambda_{\max }$ and the slit width $a=A \lambda_{\max }$ where $\lambda_{\max }$ is from the Wien displacement law. We assume that Wien's photons create a BohEmian pilot wave that contribute to the formation of the resulting pattern structure. In our experiment we should find the optimal values of D and A for the geometry of the double - slit barrier.

3) The thickness of the double - slit barrier $b=B \lambda_{\max }$ should play a significant role, too.

4) Temperature will change $\lambda_{\max }$ and should modify the intensity of the pattern structure.

5) Heraclitus' condition "Nature loves to hide" is fulfilled.

Table 3. Young - Feynman double - slit experiment for photons

$$
\begin{aligned}
& \text { Intensity of Double Slit Patterns for Photons } \\
& I=I_{0} \cos ^{2}\left(\frac{\pi d \sin \theta}{\lambda}\right)\left[\frac{\sin (\pi a \sin \theta / \lambda)}{\pi a \sin \theta / \lambda}\right]^{2} \\
& I=I_{0} \cos ^{2}\left(\frac{\pi}{\lambda} d \sin \theta\right)\left[\frac{\sin \left(\frac{\pi}{\lambda} a \sin \theta\right)}{\frac{\pi}{\lambda} a \sin \theta}\right]^{2}
\end{aligned}
$$

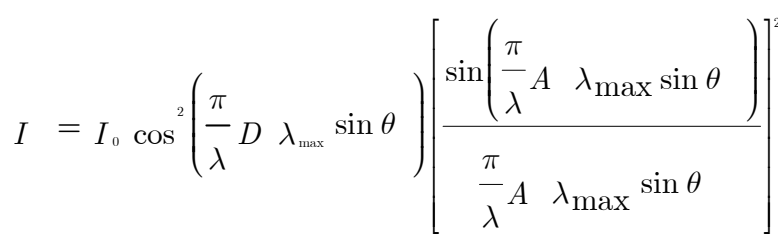

$$
\begin{aligned}
& d=D \lambda_{\max } \quad \text { separation between slits } \\
& a=A \lambda_{\max } \quad \text { slit width } \\
& \kappa=\frac{\pi}{\lambda} \quad \text { photon helical curvature, Newton }- \text { Bohm evolute } \\
& r=\frac{\lambda}{2 \pi} \quad \text { photon helical radius }
\end{aligned}
$$


The similar analysis can be done with the atom interferometry where individual atoms might react with Wien's photons. This atom interferometry experimentally opened Olivier Carnal and Jürgen Mlynek (1991) and David W. Keith with his Team (1991).

\section{Young - Feynman Double - Slit Experiment with Individual Electrons}

There were done numerous experiments to observe the pattern formation with individual electrons below the double - slit barrier. See the References.

In the Table 2 is the traditional formula for the calculation of the intensity of double slit patterns for electrons: I is the intensity at the angle $\theta, I_{0}$ is the maximum intensity, $d$ is the separation between slits, $a$ is the slit width, $\lambda$ is the pitch of the electron double helix.

We have modified this Formula in two steps:

1. We have "discovered" the hidden expression for the electron helical curvature $\kappa=2 \pi / \lambda$ - this is the hidden Bohmian pilot wave for electrons.

2. We have described the separation between slits $d$ as $d=D \lambda_{\max }$ and the slit width $a=A \lambda_{\max }$ where $\lambda_{\max }$ is from the Wien displacement law. We assume that Wien's photons create a BohEmian pilot wave that contribute to the formation of the resulting pattern structure. In our experiment we should find the optimal values of D and A for the geometry of the double - slit barrier.

3. The thickness of the double - slit barrier $b=B \lambda_{\max }$ should play a significant role, too.

4. Temperature will change $\lambda_{\max }$ and should modify the intensity of the pattern structure.

5. Heraclitus' condition "Nature loves to hide" is fulfilled.

Table 4. Young - Feynman double - slit experiment for electrons

$$
\begin{aligned}
& \text { Intensity of Double Slit Patterns for electrons } \\
& I=I_{0} \cos ^{2}\left(\frac{2 \pi d \sin \theta}{\lambda}\right)\left[\frac{\sin (2 \pi a \sin \theta / \lambda)}{2 \pi a \sin \theta / \lambda}\right]^{2} \\
& I=I_{0} \cos ^{2}\left(\frac{2 \pi}{\lambda} d \sin \theta\right)\left[\frac{\sin \left(\frac{2 \pi}{\lambda} a \sin \theta\right)}{\frac{2 \pi}{\lambda} a \sin \theta}\right]^{2} \\
& I=I_{0} \cos ^{2}\left(\frac{2 \pi}{\lambda} D \lambda_{\max } \sin \theta\right)\left[\frac{\sin \left(\frac{2 \pi}{\lambda} A\right.}{\left.\frac{2 \pi}{\max } \sin \theta\right)}\right]^{2} \\
& d=D \lambda_{\max } \quad \text { separation between slits } \\
& a=A \lambda_{\max } \quad \text { slit width } \\
& \kappa=\frac{2 \pi}{\lambda} \quad \text { electron helical curvature, Newton }- \text { Bohm evolute } \\
& r=\frac{\lambda}{4 \pi} \quad \text { electron helical radius }
\end{aligned}
$$




\section{Wilhelm Wien's Photons in the Mach - Zehnder Interferometer}

Ludwig Mach (the son of Ernst Mach) and Ludwig Zehnder in 1891 proposed the now very well-known Mach-Zehnder interferometer.

The Mach - Zehnder interferometer has been intensively studied by all scholars of quantum mechanics and is very well described in the existing literature.

In our proposal we assume that the individual Huygens - de Broglie particle reacts at the first beam-splitter with one Wilhelm Wien's photon. After that the Huygens - de Broglie particle propagates as the "sample beam" through one path while the "entangled" Wien's photon propagates as the "reference beam" through the other path. At the second beam-splitter they meet themselves again and can create the observed interference pattern at one detector.

We can experimentally test this model by inserting of filters in one or both arms of the Mach - Zehnder interferometer that are able to remove the "entangled" Wien's photons from the system while the Huygens - de Broglie particles can arrive to the detectors. In this case the Huygens - de Broglie particles should reveal their "particle behavior".

The Great New Master in the theoretical physics Paul Dirac in 1958 assumed that: "Each photon then interferes only with itself. Interference between different photons never occurs."

However, the Great New Master in the experimental quantum optics Leonard Mandel with his Team showed that two photons can interfere. See, G. Magyar and L. Mandel in 1963, R.L. Pfleeger and L. Mandel in 1967, and C.K. Hong, Z.Y. Ou and L. Mandel in 1987.

The detailed discussions to this topic were published by another Great New Masters in the experimental physics P.G. Kwiat in 2009, Markus Perner in 2013, Toshiki Kobayashi et al. in 2016, D.A. Kalashnikov et al. in 2017, D.J. Zhang et al. in 2017, and many others.

In our model the Huygens - de Broglie particles meet their Wilhelm Wien's photon partners in the first beam-splitter. After a short interaction they are separated and interacting again at the second beam-splitter and interfere with Wien's photons at the second beam-splitter. It could be another Great Surprise used by Nature to cleverly employ one of the many Wien's photons as the hidden partner for the Huygens - de Broglie particle for their joint interference. We want to pass this model into hands of Readers of this Journal better educated in Mathematics and Physics.

\section{Conclusions}

1. We have combined knowledge of Old Masters, New Masters, and Dissidents in order to newly formulate events in the Young - Feynman double- slit experiment and in the Mach-Zehnder interferometer.

2. In the classical formula for the intensity of the double-slit patterns we have identified the curvature for the Newton - Bohm evolute for photons as $\kappa=\pi / \lambda$. (Bohmian pilot wave).

3. In the classical formula for the intensity of the double-slit patterns we have identified the curvature for the Newton - Bohm evolute for electrons as $\kappa=2 \pi / \lambda$. (Bohmian pilot wave).

4. In the Young - Feynman double - slit experiment we have assumed that Wilhelm Wien's photons might contribute to the interference patter formation as the BohEmian pilot wave (Wien filter).

5. In the Mach - Zehnder interferometer we have assumed that Wilhelm Wien's photons might interact with the Huygens - de Broglie particles in the first and in the second beam-splitters.

6. We have proposed to modify those experiments by the insertion of filters to remove those Wilhelm Wien'photons and/or to change the temperature to modify the $\lambda_{\max }$ (Wien displacement law).

7. Nature might hide Her Beauty in plain sight protected by the mathematical camouflage.

8. Are there some more "hidden curves" in the Plato's Realm connected to the Photon and Electron Secrets? How to distinguish the real physical meaning written in those curves from fictious events if both are mathematically correct? How to work with the mathematical camouflage used by Nature to protect Her Secrets?

9. We want to pass this model into hands of Readers of this Journal better educated in Mathematics and Physics.

\section{Acknowledgments}

This work was supported by the JP\&FŠ Agency (Contract Number 25g/1963), by the VZ\&MŠ Agency (Contract Number 16000/1989) and by the GMS Agency (Contract Number 69110/1992). We were supported by the contract numbers $28101918 / 2018$ and 58287/2019. We have found the valuable support on the web site www.wolframalpha.com with the corrections of used formulae. 


\section{Conflict of interests}

The authors declare that there is no conflict of interests regarding the publication of this paper.

\section{References}

Ananthaswamy, A. (2019). Through Two Doors at Once. The Elegant Experiment That Captures the Enigma of Our Quantum Reality. Penguin Random House, New York, ISBN 9781101986097.

Apollonius of Perga. On the Cochlias. In L. Euclid (Ed.), Friedlein (Vol. 105, pp. 1-6).

Ashworth, R. A. (1998). Confirmation of Helical Travel of Light through Microwave Waveguide Analysis. Physics Essays, 11, 1-10. Retrieved from https://www.researchgate.net/publication/260829054 Confirmation_of_Helical_Travel_of_Light_through_Microwave_Waveguide_Analyses

Assis, A. K. T. (2003). On the First Electromagnetic Measurement of the Velocity of Light by Wilhelm Weber and Rudolf Kohlrausch. In F. Bevilacqua \& E. A. Giannetto (Eds.), Volta and the History of Electricity (pp. 267-286). Retrieved from https://www.ifi.unicamp.br/ assis/Weber-Kohlrausch(2003).pdf

Atom Interferometer. Retrieved from https://en.wikipedia.org/wiki/Atom_interferometer

Bach et al. (2013). Controlled Double-Slit Electron Diffraction. New Journal of Physics, 15, 033018.

Bardini, G., \& Gianella, G. M. (2016). A Historical Walk along the Idea of Curvature, from Newton to Gauss Passing from Euler. International Mathematical Forum, 11, 259-278. Retrieved from http://www.m-hikari.com/imf/imf-2016/5-8-2016/p/bardiniIMF5-8-2016.pdf

Batelaan et al. (2016). Momentum Exchange in the Electron Double-Slit Experiment. J. Phys. Conf. Ser., 701, 012007. Retrieved from https://pdfs.semanticscholar.org/d80e/862d3f7278e4fd7666032905 ced83ad02099.pdf

Bell, J. S. (1992). Six Possible Worlds of Quantum Mechanics. Foundations of Physics, 22, 1201-1215.

Blaschke, P. (2017). Pedal Coordinates, Dark Kepler and Other Forces Problems. Arxiv: 1704.00897v1.

Blåsjö, V. (2017). Transcendental Curves in the Leibnizian Calculus. Academic Press, Elsevier, ISBN: 978-0-12-813237-1.

Blåsjö, V. (2018). Mathematicians Versus Philosophers in Recent Work on Mathematical Beauty. Journal of Humanistic Mathematics, 8, 414-431. Retrieved from https://scholarship.claremont.edu/cgi/viewcontent. cgi?referer $=$ https: $/ /$ www.google.com/\&httpsredir $=1 \&$ article $=1380 \&$ context $=j h m$

Bohm, D. (1952). A Suggested Interpretation of the Quantum Theory in Terms of Hidden Variables, I. Physical Review, 85, 166-179.

Bohm, D. (1989). Quantum Theory. Dover Publications, ISBN-10: 9780486659695.

Böhm, J. (2016). Wonderful World of Pedal Curves. Retrieved from http://rfdz.ph-noe.ac.at/fileadmin/ Mathematik_Uploads/ACDCA/TIME2016/Boehm_Pedals_pdf

Brackenridge, J. B. (1996). The Key to Newton's Dynamics: The Kepler Problem and the Principia. Berkeley: University of California Press. ISBN: 978-0520202177.

Bruneau, O. (2015). ICT and History of Mathematics: the Case of the Pedal Curves from 17th Century to 19th Century. Retrieved from https://hal.archives-ouvertes.fr/hal-01179909/document

Carnal, O., \& Mlynek, J. (1991). Young's Double-Slit Experiment with Atoms: A Simple Atom Interferometer. Phys. Rev. Lett., 66, 2689-2692.

Cavalleri, A. (2017). Photo-induced Superconductivity. Contemporary Physics.

Circular Polarization. Retrieved from https://en.wikipedia.org/wiki/Circular_polarization

Cohen et al. (2019). Realism and Causality I: Pilot Wave and Retrocausal Models as Possible Facilitators. Arxiv: $1902.05108 \mathrm{v} 2$.

Cohen, I. B. (1999). A Guide to Newton's Principia. The Principia: The Mathematical Principles of Natural Philosophy. Berkeley, CA, University California Press. ISBN: 978-0-520-08816-0.

Cohen, I. B., \& Smith, G. E. (Eds.) (2004). The Cambridge Companion to NEWTON. Cambridge University Press, Cambridge, ISBN 0-521-65696-6.

Cook, T. A. (1914). The Curves of Life. Constable and Company, London. 
Cylindrical Helix. Retrieved from http://demonstrations.wolfram.com/SineAndCosineHelix/

Cylindrical Helix. Retrieved from http://mathworld.wolfram.com/Helix.html

Cylindrical Helix. Retrieved from https://en.wikipedia.org/wiki/Helix

Cylindrical Helix. Retrieved from https://www.mathcurve.com/courbes3d.gb/helicecirculaire/helicecirculaire. shtml

Darrigol, O. (2012). A History of Optics. From Greek Antiquity to the Nineteenth Century. Oxford University Press. Oxford. ISBN-10: 0199644373.

De Broglie - Bohm Theory. Retrieved from https://en.wikipedia.org/wiki/De_Broglie\%E2\%80\%93Bohm_theory

De Broglie, L. (1927). Electrons et Photons: Rapport et Discussions du Cinquième Conseil de Physique tenu à Bruxelles du 24 au 29 Octobre 1927. Gauthier-Villars.

De Broglie, L. (1939). Matter and light: The New Physics (pp. 136-142). W.W: Norton CO., New York.

Dennis, D. (1995). Historical Perspectives for the Reform of Mathematics Curriculum: Geometric Curve Drawing Devices and their Role in the Transition to an Algebraic Description of Functions. Dissertation at Cornell University. Retrieved from http://www.quadrivium.info/mathhistory/CurveDrawingDevices.pdf

Dirac, P. A. M. (1931). Quantised Singularities in the Electromagnetic Field. Proc. Roy. Soc. (London) A, 133, 60.

Dirac, P. A. M. (1958). Quantum Mechanics (4th ed., p. 9). Oxford Press, London.

Double-Slit Experiment. Retrieved from https://en.wikipedia.org/wiki/Double-slit_experiment

Dürer, A. (1525). Underweysung der Messung, mit dem Zirckel und Richtscheyt, in Linien, Ebenen und gantzen corporen. (Dürer's Plan for the Cylindrical Helix in a Staircase) Retrieved from https://commons.wikimedia.org/wiki/Category:Underweysung_der_Messung

Dürr, D., \& Teufel, S. (2009). Bohmian Mechanics: The Physics and Mathematics of Quantum Theory. Fundamental Theories of Physics. Springer, ISBN 10-3540893431.

Dyon. Retrieved from https://en.wikipedia.org/wiki/Dyon

Eds.) Wave - Particle Duality, Springer, ISBN 10-146136468X.

Einstein, A. (1905). Über einen die Erzeugung und Verwandlung des Lichtes betreffenden heuristischen Gesichtpunkt. Annalen der Physik, 17, 132-148.

Evans, M. W. (1993). The Photon's Magnetic Flux Quantum ВП: The Magnetic Nature of Antisymmetric Light Scattering. The Photon Magnetic Field, 227-242.

Feynman, R. (1964). Quantum Behavior. Feynman Lectures, III(1). Retrieved from http://www.feynmanlectures.caltech.edu/III_01.html

Frabboni et al. (2012). The Young-Feynman Two Slits Experiment with Single Electrons: Build-up of the Interference Pattern and Arrival-time Distribution Using a Fast-readout Pixel Detector. Ultramicroscopy, $116,73-76$.

Freire, O. (2003). Science and Exile: David Bohm, the Hot Times of the Cold War, and his Struggle for a New Interpretation of Quantum Mechanics. Retrieved from https://arxiv.org/ftp/physics/papers/ 0508/0508184.pdf

Freire, O. (2015). The Quantum Dissidents: Rebuilding the Foundations of Quantum Mechanics (1950-1990). With a Forword by S.S. Schweber. Springer, ISBN-10: 9783662446614.

Fried, M., \& Unguru, S. (2001). Apollonius of Perga's Conica: Text, Context, Subtext. Mnemosyne, Bibliotheca Classic. ISBN-10: 9004119779.

Gant, de F. (1995). Force and Geometry in Newton's Principia. Princeton University Press, Princeton. ISBN 0-691-03367-6.

Gauthier, R. (2017). Entangled Double-Helix Superluminal Composite Photon Model Defined by Fine Structure Constant. Retrieved from https://www.researchgate.net/publication/320840733_Entangled_Double-Helix_ Superluminal_Composite_Photon_Model_Defined_by_Fine_Structure_Constant

Gell-Mann, M. (1964). A Schematic Model of Baryons and Mesons. Physics Letters, 8, 214-215. 
Geminus of Rhodes: Cylindrical Helix. Retrieved from https://www-history.mcs.st-and.ac.uk/Biographies/ Geminus.html

Guicciardini, N. (2003). Reading the Principia: The Debate on Newton's Mathematical Methods for Natural Philosophy from 1687 to 1736. Cambridge University Press, Cambridge. ISBN-10: 0521544033.

Hadot, P. (2008). The Veil of Isis: An Essay on the History of the Idea of Nature. Belknap Press. ISBN-10: 0674030494.

Hall, A. R. (2015). Philosophers at War: The Quarrel Between Newton and Leibniz. Cambridge University Press. ISBN: 052152489X.

Harada et al. (2018). Interference Experiment with Asymmetric Double-Slit by Using 1.2 MV Field Emission Transmission Electron Microscope. Scientific Reports, 8, 1008.

Hardy, L. (1992). On the Existence of Empty Waves in Quantum Theory. Physics Letters A, 167, 11-16.

Hasselbach, F. (1992). Recent Contributions of Electron Interferometry to Wave - Particle Duality. In Selleri, F.

Hasselbach, F. (2010). Progress in Electron- and Ion-Interferometry. Reports on Progress in Physics, 73, 1-43.

Heat, T. L. (2015). Apollonius of Perga: Treatise on Conic Sections. Carruthers Press. ISBN-10: 1446021262.

Helical Electromagnetic Waves. (2011). Retrieved from http://blackholeformulas.com/files/EnM.html

Heron. (1912). Heronis Alexandrini opera quae supersunt omnia. Teubner, Leipzig, Ed. And translated into German by J.L. Heiberg.

Hong, C. K., Ou, Z. Y., \& Mandel, L. (1987). Measurement of Subpicosecond Time Intervals between Two Photons by Interference. Phys. Rev. Lett., 59, 2044-2046.

Hong-Ou-Mandel Effect. Retrieved from https://en.wikipedia.org/wiki/Hong\%E2\%80\%93Ou\%E2\%80\% 93Mandel_effect\#cite_note-imoto16-8

Huygens, Chr. (1678). Traité de la Lumière, Leyden, Van der Aa. (In Treatise on Light, London: McMillan, 1912).

Interpretations of Quantum Mechanics. Retrieved from https://en.wikipedia.org/wiki/Interpretations_of_ quantum_mechanics

Jönsson, C. (1961). Electroneninterferenzen an mehreren künstlich hergestellten Feinspalten. Zeitschrift für Physik A Hadrons and Nuclei, 161, 454-474.

Kalashnikov et al. (2017). Quantum Interference in the Presence of a Resonance Medium. Scientific Reports, 7, 11444. Retrieved from https://www.ncbi.nlm.nih.gov/pmc/articles/PMC5597610/

Keith et al. (1991). An Interferometer for Atoms. Phys. Rev. Lett., 66, 2693-2696.

Kobayashi et al. (2016). Frequency-Domain Hong-Ou-Mandel Interference. Arxiv: 1601.00739v1.

Köller, J. (2016). Fusspunktkurven und Gegenfusspunktkurven. Retrieved from http://www.mathematischebasteleien.de/fusspunktkurve.htm

Kvasz, L. (2012). Galileo, Descartes, and Newton - Founders of the Language of Physics. Acta Physica Slovaca, 62, 519-614. Retrieved from http://www.physics.sk/aps/pubs/2012/aps-12-06/aps-12-06.pdf

Kwiat, P. G. (2009). One- and Two-Photon Interference. In D. Greenberger, K. Hentschel, \& F. Weienrt (Eds.), Compendium of Quantum Physics. Springer Verlag, Berlin, Heidelbeerg.

Laird, W. R., \& Roux, S. (Eds.) (2008). Mechanics and Natural Philosophy before the Scientific Revolution. Springer. ISBN 978-90-481-7491-1.

Lenard, P. (1902). Über die lichtelektrische Wirkung. Annalen der Physik, 313, 149-198.

Levitt, L. S. (1978). Is the Photon a Double Helix? Lettere al Nuovo Cimento, 21, 222-223.

Lewis, P. J. (2007). Empty Waves in Bohmian Quantum Mechanics. British Journal for the Philosophy of Science, $58,787-803$.

Lockwood, E. H. (1961). A Book of Curves. Cambridge University Press. Retrieved from http://www.aproged.pt/biblioteca/ABookofCurvesLockwood.pdf

Mach, L. (1892). Über einen Interferenzrefraktor. Zeitschrift für Instrumentenkunden, 12, 89-93. 
Mach-Zehnder Interferometer. Retrieved from https://en.wikipedia.org/wiki/Mach\%E2\%80\%93 Zehnder_interferometer

Magyar, G., \& Mandel, L. (1963). Interference Fringes Produced by Superposition of Two Independent Maser Light Beams. Nature (London), 198, 255.

Mancosu, P., \& Arana, A. (2010). Descartes and the Cylindrical Helix. Historia Mathematica, 37, 403-427. Retrieved from https://www.sciencedirect.com/science/article/pii/S0315086009000949

Maor, E., \& Jost, E. (2014). Beautiful Geometry. Princeton University Press, Princeton. ISBN-13:978-0-691-15099-4.

Missiroli, G. F., Pozzi, G., \& Valdrè, U. (1981). Electron Interferometry and Interference Electron Microscopy. Journal of Physics E: Scientific Instruments, 14, 649-671.

Nauenberg, M. (1993). Newton's Early Computational Method for Dynamics. Retrieved from https://core.ac.uk/download/pdf/82476251.pdf

Nauenberg, M. (2018). Newton's Graphical Method for Central Force Orbits. Am. J. Phys., 86, 765-771.

Nauenberg, M. (2018). Visiting Newton's Atelier before the Principia, 1679-1684. Arxiv: 1805.06871v.

Negi et al. (2010). Generalization of Schwinger-Zwanziger Dyon to Quaternion. Arxiv: 1012.0279v1.

Newton, I. (1687). The Principia. Mathematical Principles of Natural Philosophy. Translated by I.B. Cohen and A. Whitman. University California Press, Berkeley. ISBN 978-0-520-08816-0.

Newton, I. (1704). Optics: or, a treatise of the reflections, refractions, inflexions, and colors of light. Also two treatises of the species and magnitude of curvilinear figures. Palo Alto, Calif:: Octavo, ISBN 1-891788-04-3.

Pedal Curves Properties. (2018). Retrieved from https://en.wikipedia.org/wiki/Pedal_curve

Perener, M. (2013). Two-Photon Interference. Retrieved from https://www.mpq.mpg.de/5020845/0508b two-photon_interference.pdf

Pfleegor, R. L., \& Mandel, L. (1967). Interference of Independent Photon Beams. Phys. Rev., 159, 1084.

Pilot Wave Theory and Quantum Realism. (2016). Space Time, PBS Digital Studios. Retrieved from https://www.youtube.com/watch?v=R1XdsyctD50

Pilot Wave Theory. Retrieved from https://en.wikipedia.org/wiki/Pilot_wave_theory

Pladevall, X. O., \& Mompart, J. (2019). Applied Bohmian Mechanics: From Nanoscale Systems to Cosmology ( $2^{\text {nd }}$ ed.). Jenny Stanford Publishing, ISBN 10-9814800104.

Plies, E., Marianowski, K., \& Ohnweiler, T. (2011). The Wien Filter: History, Fundamentals, and Modern Applications. Nuclear Instruments and Methods in Physics Research, Section A: Accelerators, Spectrometers. Detectors and Associated Equipment, 645, 7-11.

Polchinski, J. (2003). Monopoles, Duality, and String Theory. Arxiv: hep-th/0304042v1.

Roveli, C. (2018). Physics Needs Philosophy. Philosophy Needs Physics. Found. Phys., 48, 481-491. Retrieved from https://arxiv.org/ftp/arxiv/papers/1805/1805.10602.pdf

Saglam et al. (2016). Quantum Flux Associated with a Photon on the Basis of the Heisenberg Uncertainty Relation. Results in Physics, 6, 215-216. Retrieved from https://www.sciencedirect.com /science/article/pii/S2211379716300122

Saraiva et al. (2017). Photonic Counterparts of Cooper Pairs. Arxiv: 1709.04520v2

Schwinger, J. (1969). A Magnetic Model of Matter. Science, 165, 757-761.

Seager, W. (2018). The Philosophical and Scientific Metaphysics of David Bohm. Entropy, 20, 493.

Shnir, Z. M. (2005). Magnetic Monopoles (Theoretical and Mathematical Physics). Springer, ISBN-10: 3540252770 .

Stávek, J. (2017). Trigonometric Functions Above and Below of the Double-Slit Barrier. Appl. Phys. Res., 9, 59-66.

Stávek, J. (2019). Super-Elastic Double-Helix Model of Photon. Huygens-de Broglie Particle on the Helical Path Guided by the Newton-Bohm Entangled Helical Evolute. Quantum of Magnetic Flux Based on the 
Mathematical Beauty of Newton, Lorentz, Einstein, Dirac, Gell-Mann, Schwinger, Polchinski and Witten (16.06.2019). Appl. Phys. Res., 11, 40-51.

Tavabi et al. (2019). The Young-Feynman Controlled Double-Slit Electron Interference Experiment. Scientific Report, 9, 10458.

Taylor, G. I. (1909). Interference Fringes with Feeble Light. Proc. Cam. Phil. Soc., 15, 114.

Tikhonov, K. S., Skvortsov, M. A., \& Klapwijk, T. M. (2018). Superconductivity in the Presence of Microwaves: Full Phase Diagram. Physical Review B, 97, 184516.

Tombe, F. D. (2019). The 1855 Weber-Kohlrausch Experiment (The Speed of Light). The General Science Journal. Retrieved from https://www.scribd.com/document/294114501/The-1855-Weber-Kohlrausch -Experiment-The-Speed-of-Light

Tonomura et al. (1989). Demonstration of Single Electron-Buildup of an Interference Pattern. Am. J. Phys., 57, 117-120.

Walleczek, J., Grossing, G., \& Pylkkanen, P. (2019). Emergent Quantum Mechanics: David Bohm Centennial Perspectives. Mdpi AG, ISBN-10:3038976165.

Watson, J. D. (2001). The Double Helix: A Personal Account of the Discovery of the Structure of DNA. Touchstone, ISBN-10: 9780743216302.

Weinstein, G. (2015). Einstein's Pathway to the Special Theory of Relativity. Cambridge, Cambridge Scholars Publishing.

Wien Filter. Retrieved from https://en.wikipedia.org/wiki/Wien_filter

Wien, K. (1999). 100 years of Ion Beams: Willy Wien's Canal Rays. Brazilian Journal of Physics, 29, 401-413.

Wien's Displacement Law. Retrieved from https://en.wikipedia.org/wiki/Wien\%27s_displacement_law

Williams, G. (2019). Unravelling the Double Helix. The lost Heroes of DNA. Weidenfeld \& Nicholson, ISBN-10: 147460935X.

Witten, E. (1979). Dyons of Charge e $\theta / 2 \pi$. Physics Letters B, 86, 283-287.

Yates, R. C. (1974). Curves and their Properties. National Council of Teachers of Mathematics, ISBN: 10-087353039X.

Young, T. (n. d.). Retrieved from https://en.wikipedia.org/wiki/Thomas_Young_(scientist)

Zehnder, L. (1891). Ein neuer Interferenzrefraktor. Zeitschrift für Instrumentenkunden, 11, 275-285.

Zhang et al. (2017). Young's Double-Slit Interference with Two-Color Biphotons. Scientific Reports, 7, 17372. Retrieved from https://www.nature.com/articles/s41598-017-17827-8.pdf

Zweig, G. (1964). An SU(3) Model for Strong Interaction Symmetry and its Breaking. CERN Report No. 8182/TH.401.

\section{Copyrights}

Copyright for this article is retained by the author(s), with first publication rights granted to the journal.

This is an open-access article distributed under the terms and conditions of the Creative Commons Attribution license (http://creativecommons.org/licenses/by/4.0/). 